\title{
Co-Innervation of Triceps Brachii Muscle with Variant Branch of Ulnar Nerve
}

\author{
Sedat DEVELI \\ University of Health Sciences, Gulhane Faculty of Medicine, Department of Anatomy, Ankara, Turkey
}

\section{ABSTRACT}

AIM: To evaluate the existence of nerve innervation of the triceps brachii muscle via the ulnar nerve and its clinical importance in terms of nerve transfer or susceptibility to iatrogenic injuries during surgical procedures.

MATERIAL and METHODS: Thirty-five skeletally mature upper extremities of adult cadavers were included in the present study. The ulnar nerve was revealed from the medial cord to the distal part of the cubital fossa. The existence of the branches of the ulnar nerve and communicant branch of the radial nerve was examined in the brachium. The diameter and length of the variant nerve branches were measured.

RESULTS: A nerve branch from the ulnar nerve to the triceps brachii muscle was found in five of the dissected extremities (14.7\%). The mean length and diameter of the variant nerve branch were $20.2 \mathrm{~mm}$ and $1.46 \mathrm{~mm}$, respectively. The mean distance of the muscle entry point of the variant nerve branch from the bi-condylar line was $8.18 \mathrm{~cm}$.

CONCLUSION: A variant nerve branch via the ulnar nerve can innervate the medial head of the triceps brachii at the distal third of the brachium. This variant nerve branch will be under risk of iatrogenic injury during elbow surgery and it seems to be an option for nerve grafts.

KEYWORDS: Communicant branch of the radial nerve, Medial head of triceps brachii muscle, Ulnar nerve

\section{INTRODUCTION}

$\mathrm{T}$

The ulnar nerve is one the branches of the medial cord of the brachial plexus. It derives from the anterior rami of the C7-C8-T1 roots. While sensorial fibers innervate the $4^{\text {th }}$ and $5^{\text {th }}$ fingers, motor fibers innervate the hand muscles and a part of the flexor digitorum profundus muscle (9). The triceps brachii muscle is the main extensor of the elbow and it is considered to be innervated by the radial nerve, which is one of the branches of the posterior cord of the brachial plexus (9).

In recent studies, it was mentioned that in some cases, the ulnar nerve contributes the innervation of the triceps brachii muscle $(2,4,5)$. It was supposed that this kind of variation may cause susceptibility to iatrogenic injuries, especially during posterior surgical interventions on the distal humerus (5). In addition, it was suggested that these variant nerve branches could be useful for nerve transfer in brachial plexus injuries (4). However, conversely, some authors stated that the ulnar nerve does not have any branch in the upper arm (6).

The present study aimed to evaluate the existence of nerve innervation of the triceps brachii muscle via the ulnar nerve and its clinical importance in terms of nerve transfer or susceptibility to iatrogenic injuries during surgical procedures, in Anatolian adult cadavers.

\section{MATERIAL and METHODS}

A total of 35 skeletally mature upper extremities of adult cadavers, obtained from the Department of Anatomy, were 
included in the present study. The cadavers were fixed with formaldehyde and had belonged to Caucasian subjects. The ages of the cadavers were unknown. One extremity was excluded from the study due to humerus fracture. This study was conducted with the ethical approval of the local ethics committee (Approval number 2016-13).

With the cadaver in the prone position, the olecranon and medial epicondyle of the humerus were marked over the skin (Figure 1A). A vertical skin incision was made from the olecranon to the shoulder region. The skin and superficial fascia were opened with blunt dissections. The superficial veins and cutaneous nerves of the arm were revealed and retracted in the dissected area (Figure 1B). Subcutaneous fatty tissue was removed with blunt dissections, and the triceps brachii muscle and its deep fascia were revealed. The ulnar nerve was identified at the anterior of the medial head of the triceps brachii muscle, and its course was revealed from distal to proximal (Figure 1C). The fibrous tissue covering the ulnar nerve was removed in order to reveal the nerve (Figure 1D). Existence of the branches of the ulnar nerve in the brachium and communicant branch of the radial nerve (r.communicans ulnaris) were examined in the dissected area. The distance between the uppermost edge of the upper extremity and the tip of the olecranon was measured as the length of the upper arm. These reference points are also surface markings of the upper extremity and can easily be detected by physical examination or in surgery. The diameter and length of the ulnar nerve branches to the triceps brachii muscle were measured, and the distance of these branches to the imaginary line passing through the short axis of the condyles of the distal humerus (bi-condylar line) was noted. In order to reveal the whole innervation pattern of the triceps brachii muscle, in the existence of the branch of the ulnar nerve innervating the triceps brachii muscle, the branches of the radial nerve to the muscle were also revealed. All specimens were digitally photographed and the measurements were made on images with the help of the image processor program ImageJ with guidance of the scale-bar previously placed next to samples. A Canon IXUS 115 digital camera was used for photography.

Descriptive analyses were made for all variables. Statistical results are represented as the mean \pm standard deviation (SD). Student's t-test was performed to compare the measurements of the left and right sides. All analyses were done using the SPSS (version 15) software with statistical significance set at $\mathrm{p}<0.05$.

\section{RESULTS}

The mean length of the upper arm was found to be $34.31 \mathrm{~cm}$ on the right side and $34.15 \mathrm{~cm}$ on the left side. A nerve branch from ulnar nerve to triceps brachii muscle was found in five of the dissected extremities (14.7\%) (Figure 2). Three of the variant nerve branches were on the right side, and two were on the left side. Bilateral variant nerve branching was found
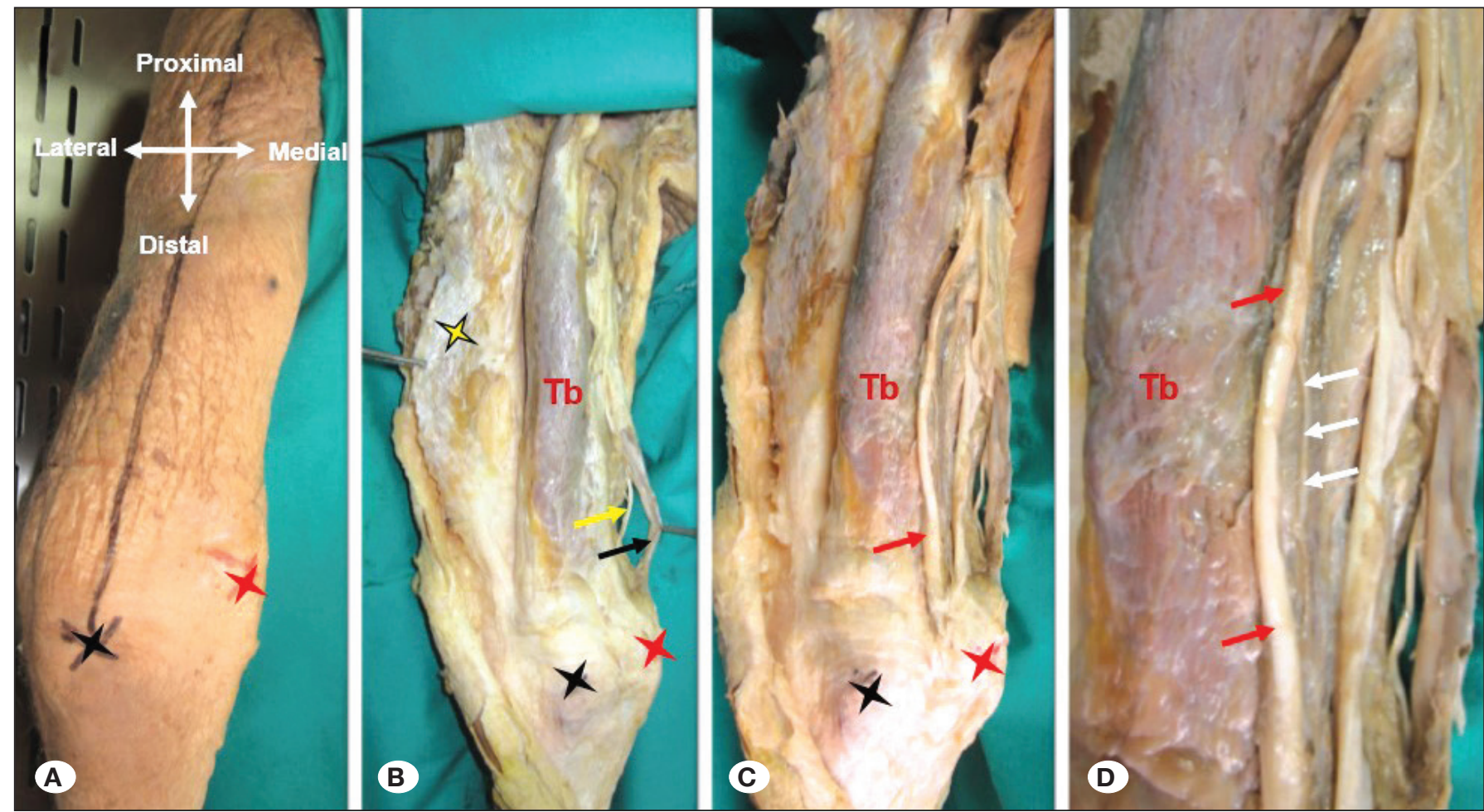

Figure 1: Dissection of the upper arm. A) Marking of the surface points. B) Removal of skin and superficial fascia. C) Reveal of triceps brachii muscle and ulnar nerve. D) Examination of ulnar nerve. Black mark: Olecranon, Red mark: Medial epicondyle of humerus, Yellow mark: Superficial fascia, Black line: Vertical incision line, Yellow arrow: Cutaneous nerve, Black arrow: Superficial vein, Red arrow: Ulnar nerve, White arrow: Variant nerve branch. Tb: Triceps brachii muscle. 
in one cadaver. All these nerve branches were innervating the medial head of the triceps brachii (Figure 3). Mean length and diameter of the variant nerve branch were $20.2 \mathrm{~mm}$ and 1.16 $\mathrm{mm}$, respectively. Meanwhile, the mean distance of the muscle entry point of the variant nerve branch from the bi-condylar line was $8.18 \mathrm{~cm}$. Given our measurements, the variant nerve

Table I: Morphometric Data of the Variant Nerve Branch

\begin{tabular}{|c|c|c|}
\hline Parameters & \multicolumn{2}{|c|}{ Mean } \\
\hline \multirow{2}{*}{ Length of upper arm } & Right & Left \\
\hline & $34.31 \mathrm{~cm}$ & $34.15 \mathrm{~cm}$ \\
\hline Length of variant nerve branch & \multicolumn{2}{|c|}{$2.02 \mathrm{~cm}$} \\
\hline Diameter of variant nerve branch & \multicolumn{2}{|c|}{$1.46 \mathrm{~mm}$} \\
\hline Diameter of ulnar nerve & \multicolumn{2}{|c|}{$5.34 \mathrm{~mm}$} \\
\hline
\end{tabular}

Distance of muscle entry point of variant nerve branch from $8.18 \mathrm{~cm}$ bi-condylar line branch is observed on the distal one-third of the upper arm. Morphometric data are given in Table I.

In one of the other extremities, a communicating branch between the radial nerve and ulnar nerve was also found (Figure 4). The branching point of the communicating branch from the radial nerve was $21.3 \mathrm{~cm}$ proximal to the bi-condylar line and it was joining the ulnar nerve $16.3 \mathrm{~cm}$ proximal to the bi-condylar line.

The innervation pattern of the triceps brachii muscle from the radial nerve was also revealed in the existence of the variant nerve branch from the ulnar nerve. The lateral head of the triceps brachii muscle was innervated by two branches of the radial nerve in 3 of 5 extremities and by one branch of the radial nerve in 2 of 5 extremities. The long head of the triceps brachii muscle was innervated by one branch of the radial nerve in all 5 of the extremities. Examining the innervation pattern of the medial head of the triceps brachii muscle showed that in 3 of the extremities $(60 \%)$ the radial nerve was also giving a motor branch to the medial head, besides the variant nerve branch from the ulnar nerve. The mean distance from the bi-condylar line of these 3 branches was $14.1 \mathrm{~cm}$.
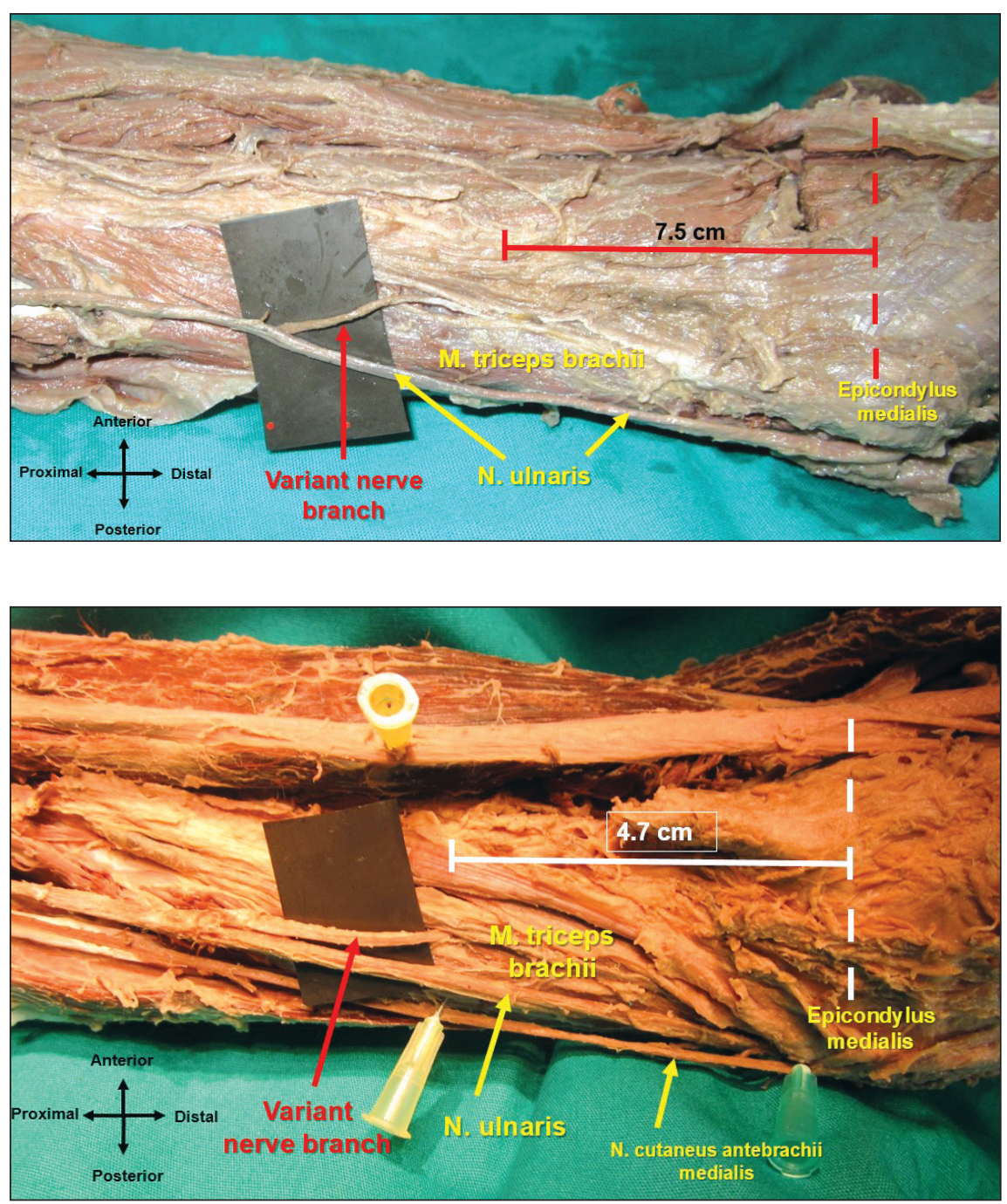

Figure 2: Medial view of the left arm. Variant nerve branch innervating triceps brachii muscle via ulnar is seen in the distal third of the brachium (red arrow).
Figure 3: Anteromedial view of the left arm. Variant nerve branch of the ulnar nerve is seen (red arrow). N.cutaneus antebrachii medialis was reflected. 


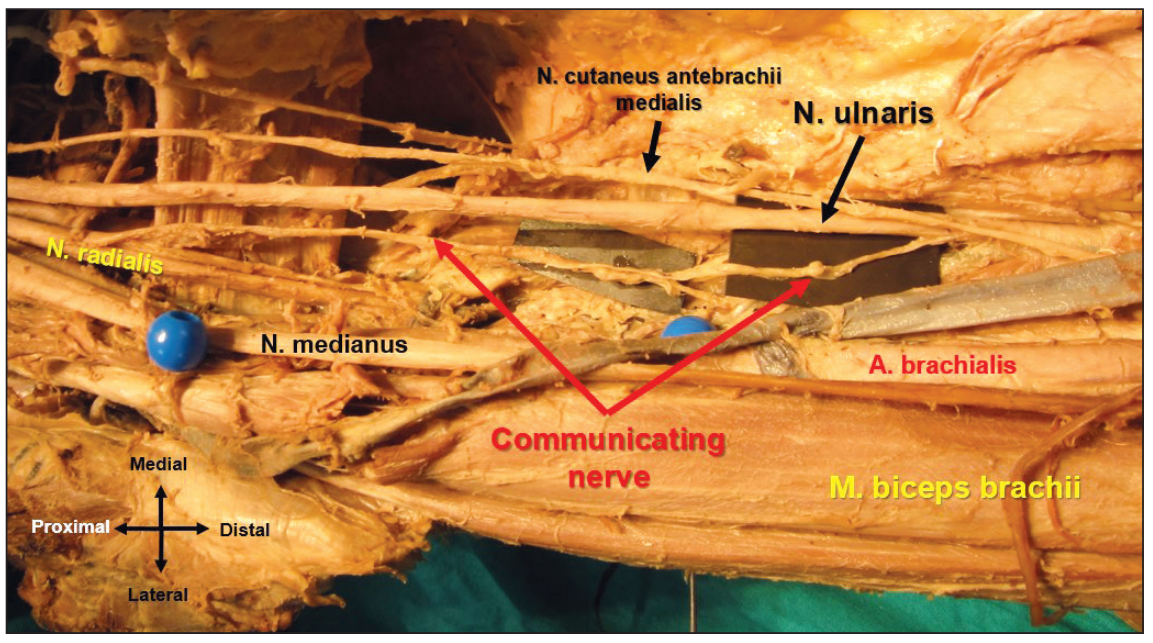

Figure 4: Anterior view of the right arm. Communicating branch between radial and ulnar nerves is seen (red arrows). Note that radial nerve gives off the communicating branch in the upper third of the brachium.
No statistically significant difference was found between the measurements of the right and left sides ( $p>0.05)$.

\section{DISCUSSION}

Besides gross anatomy, the anatomical variations of the peripheral nerves have clinical importance in terms of iatrogenic injuries during surgical procedures and for interpreting electrophysiological tests for entrapments or nerve transfer procedures. In normal anatomy, the ulnar nerve is described as the largest branch of the medial cord of the brachial plexus and does not have any branch in the upper arm. However, there are several reported variations of the nerves of the upper extremity, such as the formation of the median nerve or dual innervation of muscles such as the brachialis muscle.

In recent studies, the ulnar nerve was reported to contribute to the innervation of the triceps brachii muscle $(2,4,5,8)$. Bekler et al. have reported that in 11 of $18(61 \%)$ extremities the ulnar nerve had a branch in the medial head of the triceps brachii muscle (2). Loukas et al. described that in 14 of 50 (28\%) arms, the ulnar nerve contributed to the innervation of the medial head (4). Silva et al. found this variation in 9 of 60 (15\%) extremities (8). In our study, we found an incidence of $14.7 \%$ from 34 extremities. Our results show a lower percentage relative to the literature.

Silva et al. found variant nerve branches in the distal and middle segments of the arm (8). Loukas et al. observed that variant branches of the ulnar nerve were extending in the level of the lower quadrant of the humerus, and Bekler et al. reported that these branches were proximate to the medial epicondyle $(2,4)$. In a case report, the variant nerve branch was reported to be branching from the ulnar nerve $10 \mathrm{~cm}$ proximal to the elbow (5). The variant nerve branches was observed in the lower one-third of the upper arm, which is consistent with the literature. However, branches coursing in the middle segment of the upper arm no were idetified.

In the North American population, the mean length and diameter of the variant nerve were reported as $3.4 \mathrm{~mm}$ and $0.9 \mathrm{~mm}$, respectively (4). In the South American population, the length of the variant nerve was reported to be $3.72 \mathrm{~cm}$ and the diameter of the nerve was observed as less than 1 $\mathrm{mm}$ (8). According to the results of this study, in Turkish population, the length of the variant nerve is longer than that in the North American population and shorter than that in the South American population. However, the diameter of the variant branch was found to be wider than both other studies. The variant nerve branches observed in our samples were not as twigs mentioned in previous studies (less than $1 \mathrm{~mm}$. in diameter). The diameter and the length of the variant nerve branch (Table I) seem to be sufficient for nerve transfers such as thenar branches of the median nerve or anterior-posterior interosseous nerves $(1,10)$. The postoperative motor deficit of the medial head of the triceps brachii will be less pronounced due to dual innervation from the radial nerve in terms of nerve transfers.

On the other hand, as also mentioned in classic anatomy textbooks, some authors stated that the ulnar nerve does not have any branch in the brachium, and thus that the entire innervation of the triceps brachii muscle is supplied by the radial nerve $(3,6,7)$. In a recent study, the nerve branch from the ulnar nerve to the medial head of the triceps brachii muscle was considered as the ulnar collateral branch of the radial nerve coursing within the nerve sheet of the ulnar nerve (6). In Nomina Anatomica, no ulnar collateral branch of the radial nerve was mentioned. Thus, we assume that the authors intended to refer to the communicating branch of the radial nerve with the ulnar nerve (r.communicans ulnaris). In their study, the authors state that this communicating branch was observed in all specimens, and it was reported to be joining the ulnar nerve in the proximal one-third of the brachium. Fibers of the communicating nerve were found to be coursing without intermingling with the fibers of the ulnar nerve and were emerging from the ulnar nerve to the muscle in the distal third of the brachium (6). As a result of their study, the authors state that roots of the variant nerve belong to the radial nerve, but it courses within the nerve sheet of the ulnar nerve (6).

In the light of the literature, it has been seen that the debate about the variant nerve branch depends on the roots of the nerve. Some authors state that the nerve branch from ulnar 
nerve to the medial head of the triceps brachii muscle is the communicant branch of the radial nerve (r.communicans ulnaris) $(3,6)$, while some authors state that this nerve is a branch of ulnar nerve $(2,4,5,8)$. In the present study, the aim was not to reveal the roots of the variant nerve branch, but to expand the morphometric knowledge in terms of surgical interventions of the elbow or nerve transfer procedures.

R.communicans ulnaris is a branch of the radial nerve and mostly seen in the proximal third of the brachium. In the present study, this communicating branch was found in one sample and no branch via ulnar nerve to triceps brachii in this sample was observed. In Pascual-Fonts' study, this communicating branch was observed in all fetal samples (6). We think that this difference may be a result of the samples, since our study was conducted on adult cadavers.

On the other hand, our results indicate that medial head of the triceps brachii has a dual innervation in $60 \%$ of the samples, in the existence of the variant nerve branch from the ulnar nerve. As far as we know, there is no reported data on dual innervation of the medial head of the triceps brachii in the literature.

\section{CONCLUSION}

Both the literature data and the findings of the current study show that a variant nerve branch via the ulnar nerve can innervate the medial head of the triceps brachii at the distal third of the brachium. Although the roots of this variant nerve are controversial, this variant nerve branch will be under risk of iatrogenic injury during posterior or medial approaches to the elbow. In addition, entrapment of the nerve by compression, slapping, rubbing, or slipping can be seen. In this case, interpreting electrophysiological tests and radiological images becomes important. Furthermore, this nerve will be another option for nerve grafts.

\section{REFERENCES}

1. Baltzer $\mathrm{H}$, Woo A, Oh $\mathrm{C}$, Moran SL: Comparison of ulnar intrinsic function following supercharge end-to-side anterior interosseous-to-ulnar motor nerve transfer: A matched cohort study of proximal ulnar nerve injury patients. Plast Reconstr Surg 138: 1264-1272, 2016

2. Bekler H, Wolfe VM, Rosenwasser MP: A cadaveric study of ulnar nerve innervation of the medial head of triceps brachii. Clin Orthop Relat Res 467: 235-238, 2009

3. Cruveilhier J: Descriptive Anatomy. Vol: 4. Paris: Bechet Jeune, 1836:809-816

4. Loukas M, Bellary SS, Yuzbasioglu N, Shoja MM, Tubbs RS, Spinner RJ: Ulnar nerve innervation of the medial head of the triceps brachii muscle: A cadaveric study. Clin Anat 26: 10281030, 2013

5. Miguel-Perez MI, Combalia A, Arandes JM: Abnormal innervation of the triceps brachii muscle by the ulnar nerve. $J$ Hand Surg Eur Vol 35: 430,431, 2010

6. Pascual-Font A, Vazquez T, Marco F, Sanudo JR, RodriguezNiedenfuhr M: Ulnar nerve innervation of the triceps muscle: Real or apparent? An anatomic study. Clin Orthop Relat Res 471: 1887-1893, 2013

7. Ramström M: Investigations on the innervation of the medial head of the triceps brachii muscle. Anat Anz 19:420-431, 1918

8. Silva D, Barros M, Freire T, Júnior LF, Almeida Filho W, Cadeira JC, Silva N: Ulnar nerve contribution in the innervation of the triceps brachii muscle ulnar nerve to the triceps brachii. Anatomy Journal of Africa 6: 834-839, 2017

9. Standring S: Gray's anatomy: The anatomical basis of clinical practice. New York: Elsevier Limited, 2016

10. Xu B, Dong Z, Zhang CG, Gu YD: Multiple nerve and tendon transfers: A new strategy for restoring hand function in a patient with C7-T1 brachial plexus avulsions. J Neurosurg 127: 837-842, 2017 